\title{
Zinc oxide as an ozone sensor
}

\author{
R. Martins, E. Fortunato, P. Nunes, I. Ferreira, and A. Marques \\ Department of Materials Science/CENIMAT, Faculty of Sciences and Technology, \\ New University of Lisbon and CEMOP/UNINOVA, 2829-516 Caparica, Portugal \\ M. Bender \\ Applied Films GmbH \& Co. KG. Siemensstrasse 100, 63755 Alzenau, Germany \\ N. Katsarakis, V. Cimalla, and G. Kiriakidis \\ Photonic and Electronic Materials Lab, Institute of Electronic Structure and Laser, \\ Foundation for Research and Technology - Hellas, P. O. Box 1527, 71110 Heraklion, Crete, Greece
}

(Received 7 January 2003; accepted 4 May 2004)

\begin{abstract}
This work presents a study of intrinsic zinc oxide thin film as ozone sensor based on the ultraviolet (UV) photoreduction and subsequent ozone re oxidation of zinc oxide as a fully reversible process performed at room temperature. The films analyzed were produced by spray pyrolysis, dc and $\mathrm{rf}$ magnetron sputtering. The dc resistivity of the films produced by $\mathrm{rf}$ magnetron sputtering and constituted by nanocrystallites changes more than eight orders of magnitude when exposed to an UV dose of $4 \mathrm{~mW} / \mathrm{cm}^{2}$. On the other hand, porous and textured zinc oxide films produced by spray pyrolysis at low substrate temperature exhibit an excellent ac impedance response where the reactance changes by more than seven orders of magnitude when exposed to the same UV dose, with a response frequency above $15 \mathrm{kHz}$, thus showing improved ozone ac sensing discrimination. (C) 2004 American Institute of Physics. [DOI: 10.1063/1.1765864]
\end{abstract}

\section{INTRODUCTION}

The growing demand for gas sensing devices to monitor and to control environmental conditions, such as the presence of ozone or carbon monoxide in the atmosphere, made zinc oxide $(\mathrm{ZnO})$ highly attractive for this application due to its high sensitivity to many gases and availability for preparation by many reliable processes. Most of them use, in its bulk form, ${ }^{1,2} \mathrm{ZnO}$, a wide band gap semiconductor $\left[E_{\text {gap }}\right.$ $\sim 3.2 \mathrm{eV}$ (Ref. 3)] that can also be utilized in other applications, such as thin film transistors, ${ }^{4}$ surface acoustic wave devices, optical wave guides, ${ }^{5}$ varistors ${ }^{6}$ and as a front contact in optoelectronic devices. ${ }^{7}$

The recent improvement of doped and undoped $\mathrm{ZnO}$ films prepared by various methods with the required gas sensitivity and selectivity of hydrocarbons, ammonia, oxygen, and $\mathrm{NO}_{x}$ has contributed to the extensive study of this material as a gas sensor. ${ }^{8-10}$ The authors of this work would like to emphasize the recent trend to develop gas sensors using thin film technology, since the gas sensing principle is associated to a surface phenomenon rather than to a bulk phenomenon. ${ }^{11,12}$

To date, the use of oxide metal films as ozone sensors required an active heating element and subsequent operations at high temperatures (above $573 \mathrm{~K}$ ), demanding a highenergy consumption for this type of sensor elements. ${ }^{13,14}$ To overcome this difficulty, we have demonstrated that the conductivity of some of these oxides can change orders of magnitude after exposition to UV radiation and subsequent oxidation, allowing them to work as ozone sensors at room temperature. 15,16

In the present work ac impedance measurements ${ }^{17-19}$ were introduced before and after UV irradiation, followed by ozone exposition, to locally try to measure and to separate the role of grains, surface grains, grain boundaries, interfaces, and electrodes on the type of sensor response obtained. ${ }^{20}$ As examples, we can mention the one observed in $\mathrm{ZnO}$ and $\mathrm{ZnO}$ Bi doped used to produce varistors, ${ }^{21-23}$ or the behavior observed in semiconducting ceramics such as $\mathrm{Ba} \mathrm{TiO}_{3}$, electrical inhomogeneous materials. ${ }^{24}$

In addition to the ac sensing properties, we also analyzed the dc sensing behavior towards UV and ozone of the undoped $\mathrm{ZnO}$ films produced by different techniques.

\section{EXPERIMENTAL DETAILS}

For this work, $\mathrm{ZnO}$ films were deposited onto soda-lime glass substrates by different techniques [dc and rf magnetron sputtering $\left(\mathrm{Sp}_{\mathrm{rf}}\right)$, and spray pyrolysis $\left.(\mathrm{SP})\right]$. The dc sputtering films were made at substrate temperatures $T_{s}$ ranging from room temperature up to $725 \mathrm{~K}$, using a deposition pressure of $\approx 1 \mathrm{~Pa}$ and a background pressure of about $6.7 \times 10^{-5} \mathrm{~Pa}$. The films deposited by SP, $T_{s}$ varied from 523 to $775 \mathrm{~K}$, using the procedures described later in this work. ${ }^{25}$ For the $\mathrm{Sp}_{\mathrm{rf}}$ films, $T_{s}$ were fixed at room temperature and the deposition pressure varied between 1.06 and $2.93 \mathrm{~Pa}$, using the procedure described elsewhere. ${ }^{26}$ All sputtered films had thicknesses measured by the stylus method of about $200 \mathrm{~nm}$, except the films deposited by SP which, in some cases, had thicknesses of about $2 \mu \mathrm{m}$.

The dc and ac films' conductivities were measured using evaporated $\mathrm{NiCr}$ contacts in a coplanar gap $(1.5 \mathrm{~mm})$ configuration. Their sensitivity was determined by irradiating them with an ultraviolet (UV) light [mercury pencil lamp from Edmund Scientific having an average intensity of $4 \mathrm{~mW} / \mathrm{cm}^{2}\left(0.5 \mathrm{~mW} / \mathrm{cm}^{2}\right.$ for the ac measurements $)$ at a wavelength of $254 \mathrm{~nm}$ ] for $20 \mathrm{~min}$. They were then subjected to oxidation in ozone for $50 \mathrm{~min}$, with online dc and 
ac impedance monitoring, using a chamber described elsewhere, ${ }^{27}$ where the backing pressure was of $1.06 \mathrm{~Pa}$. For the dc conductivity measurements, a 617 Keithley electrometer computer controlled was used. The ac impedance measurements were performed using a precision impedance analyzer (Agilent $4294 \mathrm{~A}$ ) in the frequency range from $40 \mathrm{~Hz}$ to $110 \mathrm{MHz}$ in 201 steps, before and after UV exposition, using the same electrodes and configuration described above. Here, each measurement corresponded to the average value taken over 20 measurements with a backing pressure of $0.32 \mathrm{~Pa}$. This procedure allowed us to decrease the error ascribed to each measurement and to avoid problems related to surface conductions due to air moisture dependent on environmental conditions, when measurements are not performed under vacuum conditions. In addition, the impedance behavior of the substrate was tested to see if the substrate played any role on the set of results achieved. The data showed us that the substrate had no influence on the set of results obtained.

The ozone sensitivity of these films was determined by controlling the ozone pressure within the chamber. With the existing setup, however, it was not possible to determine the amount of generated ozone. The only data that we have is the ratio of the backing pressure to the oxygen pressure inside the chamber that was exposed to UV to produce ozone. Thus, for the production of ozone for testing the dc and ac sensitivity to ozone of the films, the chamber was, respectively, backfilled with oxygen at pressures of 59.85 and $49.8 \mathrm{~Pa}$ and then exposed to UV treatment using the power densities indicated above. During this procedure, the sample was shielded from the UV light.

The films studied exhibit different types of structures (ranging from polycrystalline to nanocrystalline), surface morphologies, and degrees of porosity which were analyzed, respectively, by x-ray diffraction (XRD), secondary electron microscope (SEM), and atomic force microscopy (AFM). The objective was to correlate the characteristic features obtained with the type of ac and dc sensitivity behavior observed.

\section{RESULTS}

\section{A. Structure and morphology}

The XRD data achieved (Fig. 1) show that all films produced were polycrystalline or nanocrystalline (crystallite sizes below $50 \mathrm{~nm}$ ), either c-axis oriented or random oriented. The XRD patterns also reveal the existence of a single phase with a hexagonal wurtzite structure. Here, the alternating layers of $\mathrm{Zn}$ and $\mathrm{O}$ atoms are distributed in such a way that each type of atoms occupies the center of a tetrahedron in whose vertices the atoms of the other element ${ }^{28}$ of bulk are located. In the case of $\mathrm{Sp}_{\mathrm{rf}}$ film, only the 002 orientation is observed. This means that $\mathrm{Sp}_{\mathrm{rf}}$ films are strongly textured, as also observed by other investigators. ${ }^{29}$ The films deposited by SP exhibit a random oriented structure that changes strongly with the substrate or annealing temperature used.

For films produced by de sputtering, a $z$-axis oriented growth is observed with a decrease of film stress for the more stoichiometric films, as revealed by the decrease of the derived lattice constants. Grain size and lattice constants, de-
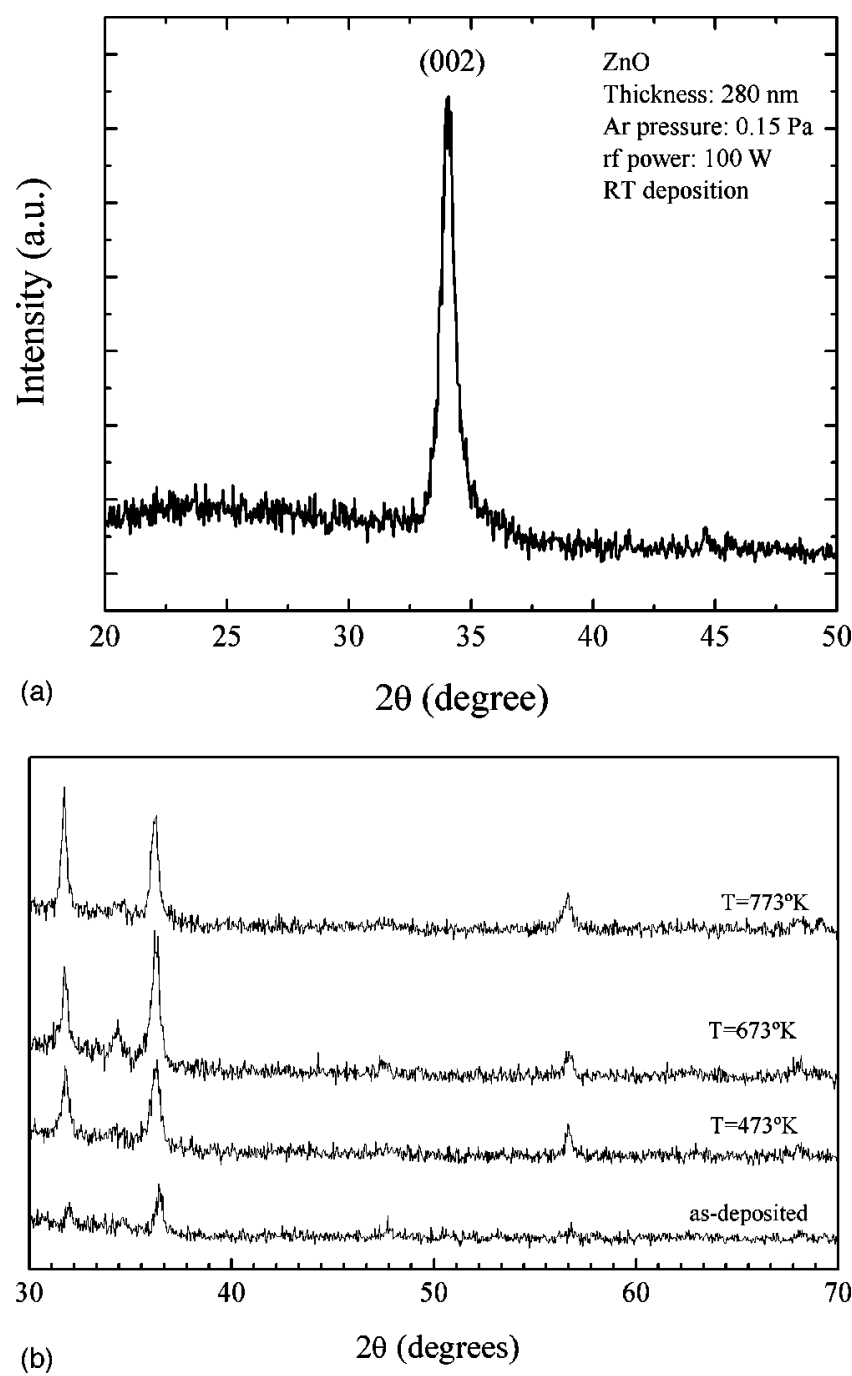

FIG. 1. XRD patterns of undoped $\mathrm{ZnO}$ films produced by spray pyrolysis and annealed at different temperatures as shown at the inset (a); a rf magnetron sputtering film (b).

rived from the XRD data, reveal that the grain size (determined by the Scherrer formula ${ }^{30}$ ) enhances as $T_{s}$ increases: The highest grain size is achieved for films produced by SP (Ref. 31) whose size increases with the annealing temperature.

The SEM and AFM analysis allowed us to study the morphology of the films produced, to infer the size of the grains/clusters formed, and to analyze possible defects existing between grains (see Fig. 2). The data achieved reveal films with different surface morphologies and grain sizes. The SEM data agree with the surface images obtained by AFM, showing SP films with a high surface roughness and high grain sizes or $\mathrm{Sp}_{\mathrm{rf}}$ films with small grains constituted by nanocrystals.

The films with the highest degree of roughness show grains with irregular shapes and dimensions of the order of micrometers (films produced by SP or dc sputtering at high $T_{s}$ ). Less rough films show a surface covered with regular shaped grains, with dimensions of some tens of nanometers (films produced by dc or rf sputtering at low $T_{s}$ ). Here, we can visibly notice that the surfaces of the $\mathrm{Sp}_{\mathrm{rf}}$ films are less 


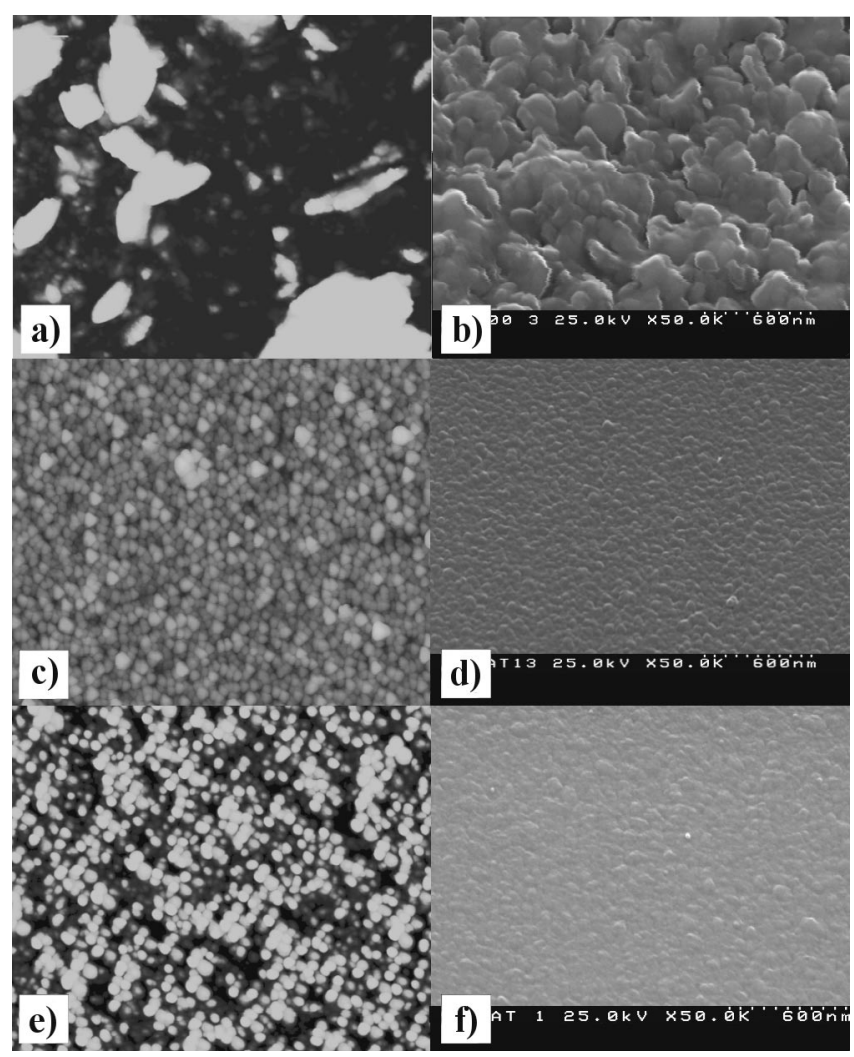

FIG. 2. AFM surface images and SEM micrographs of undoped $\mathrm{ZnO}$ films produced by spray pyrolysis (a), (b) with $2 \mu \mathrm{m}$ grains and dc sensitivity of 6.7 : dc sputtering (c), (d) with $70 \mathrm{~nm}$ grains and dc sensitivity of $4.5 \times 10^{4}$; rf sputtering (e), (f) with $87 \mathrm{~nm}$ grains and dc sensitivity sensor of 3.1 $\times 10^{4}$. For the AFM images the area shown is $5 \times 5 \mu \mathrm{m}^{2}$ with a $z$ scale of $500 \mathrm{~nm}$ (a), and $2 \times 2 \mu \mathrm{m}^{2}$ with a $z$ scale of $50 \mathrm{~nm}$ (c), (e), respectively. The scale for the SEM photographs is indicated inside the figures (b), (d), and (f).

rough and more compact and dense than the SP films that are highly porous with a large number of surface defects and contaminants, mainly when $T_{s}$ used is low.

The lateral grain size, the rms roughness, and the effective surface were extracted from the AFM data. The effective surface of the samples analyzed was found to be constant for all films. The rms roughness was estimated to be between 10 and $100 \mathrm{~nm}$ for the films deposited by SP, decreasing to values of $1-10 \mathrm{~nm}$ for the sputtered films.

\section{B. Electrical measurements: ac and dc conductivity}

For the set of films produced we analyzed the behavior of the real $\left(Z^{\prime}\right)$ and imaginary $\left(-Z^{\prime \prime}\right)$ components of the impedance $(Z)$ for $\mathrm{SP}$ and $\mathrm{Sp}_{\mathrm{rf}}$ films with the frequency $(f)$. Figures 3 and 4 show the behavior of $Z^{\prime}$ and $-Z^{\prime \prime}$ with $f$, before and after UV irradiation (for $15 \mathrm{~min}$ ), under a vacuum of $0.32 \mathrm{~Pa}$, followed by the exposition of the films to an ozone atmosphere for about $20 \mathrm{~min}$ at a pressure of $49.8 \mathrm{~Pa}$. The data in Fig. 3 show that the noise has very little influence on the data recorded. The dependence of $Z^{\prime}$ on $f$ is highly dependent on the structure of the films analyzed and environmental conditions used.

For the SP films, we can see that $Z^{\prime}$ is $f$ independent up to frequencies of about $15-25 \mathrm{kHz}$, depending on $T_{s}$ used (see Table I). Above this, $f Z^{\prime}$ decreases linearly as $f$ in- creases, in the log-log plot shown. The frequency above which $Z^{\prime}$ is reduced by $2^{1 / 2}$, which we call cutoff frequency $\left(f_{c}\right) . f_{c}$ is highly enhanced (from one to about three orders of magnitude) after the film is exposed to UV irradiation, and the function of the type of structure and morphology of the films analyzed. After exposing the films to an ozone atmosphere, $f_{c}$ decreases, as shown in Table I. In the limit, after exposing the films to a $10^{5} \mathrm{~Pa}$ ozone pressure, $f_{c}$ recovers its initial value. The ratio of $Z^{\prime}$ before and after UV $\left(S_{\mathrm{UV}}^{\prime}\right)$ irradiation gives us a good indication of how sensitive the films are to UV irradiation. The data show that $S_{\mathrm{UV}}^{\prime}$ varies from about four orders of magnitude to about a factor of 15 as $T_{s}$ increases from 598 to $648 \mathrm{~K}$. That is, the less porous films exhibit the lowest $S_{\mathrm{UV}}^{\prime}$.

For the $\mathrm{Sp}_{\mathrm{rf}}$ films analyzed-sample 7 has a grain size of about $30 \mathrm{~nm}$ and sample 10 has a grain size of about $55 \mathrm{~nm}-f_{c}$ is located, respectively, at 2 and $10 \mathrm{kHz}$, where the highest change of $Z^{\prime}\left(S_{\mathrm{UV}}^{\prime}=5 \times 10^{4}\right)$ occurs for the films with the lowest grain size.

For all films analyzed we have also noticed that, after exposing the films to an ozone atmosphere, $Z^{\prime}$ increases monotonically with the pressure of ozone used, recovering its initial impedance at a pressure of about $10^{5} \mathrm{~Pa}$.

Figure 4 shows the dependence of $-Z^{\prime \prime}$ on $f$, revealing a high noise contribution for the low frequency range. This behavior is attributed to the type of geometry used and the high resistance of the films. The $-Z^{\prime \prime}$ data of the SP films obtained before UV irradiation and under vacuum conditions $(0.32 \mathrm{~Pa})$ show a differently located peak, the function of the degree of the films' compactness, the surface roughness, and crystals size. The film deposited at $T_{s}=598 \mathrm{~K}$ has the lowest grain size. It is highly rough and porous, exhibiting a sensitivity $\left(-Z^{\prime \prime}\right.$ ratio before and after UV irradiation) $S_{\mathrm{UV}}$ at $f_{\text {peak }}=15 \mathrm{kHz}$ of about $5.5 \times 10^{7}$, under a pressure of $0.32 \mathrm{~Pa}$. After exposing the films to an ozone pressure (49.8 Pa), the peak shifts towards $170 \mathrm{kHz}$ and the corresponding ozone sensitivity $S_{\mathrm{O}}$ becomes about $2.5 \times 10^{5}$. For films produced at $T=623 \mathrm{~K}$, the grain size increases while the surface roughness decreases, but the film is still porous. The $f_{\text {peak }}$ is around $24 \mathrm{kHz}, S_{\mathrm{UV}}=10^{6}$ and $S_{\mathrm{O}}=90$. For films produced at $T=648 \mathrm{~K}$, the grain size and defects between grains are high, as revealed by SEM analysis. $f_{\text {peak }}$ $\approx 22 \mathrm{kHz}$. It is shifted to $130 \mathrm{kHz}$ after UV/ozone exposition to which it corresponds to $S_{\mathrm{UV}}=10^{4}$ and $S_{\mathrm{O}}=1.9$, as shown in Table I.

Overall, we are able to observe that $f_{\text {peak }}$ coincides with $f_{c}$ obtained from the $Z^{\prime}$ plots as a function of $f$. However, the corresponding $S_{\mathrm{UV}}$ and $S_{\mathrm{O}}$ sensitivities are now higher than the ones obtained from $Z^{\prime}$. This could mean that the surface dielectric relaxation losses ${ }^{17,32}$ play an important role here.

The main feature for the $\mathrm{Sp}_{\mathrm{rf}}$ films is that the $f_{\text {peak }}$ (or $f_{c}$ ) does not change after UV irradiation or of exposure of the films to an ozone atmosphere. The $f_{\text {peak }}$ recorded are smaller than the ones reported for the SP films. This behavior is attributed to the fact that the $\mathrm{Sp}_{\mathrm{rf}}$ films are less porous and surface contaminated than the SP films. For these films, we also notice that $S_{\mathrm{UV}}^{\prime}$ is higher than the values recorded for SP films with similar grain sizes, while the same does not hap- 

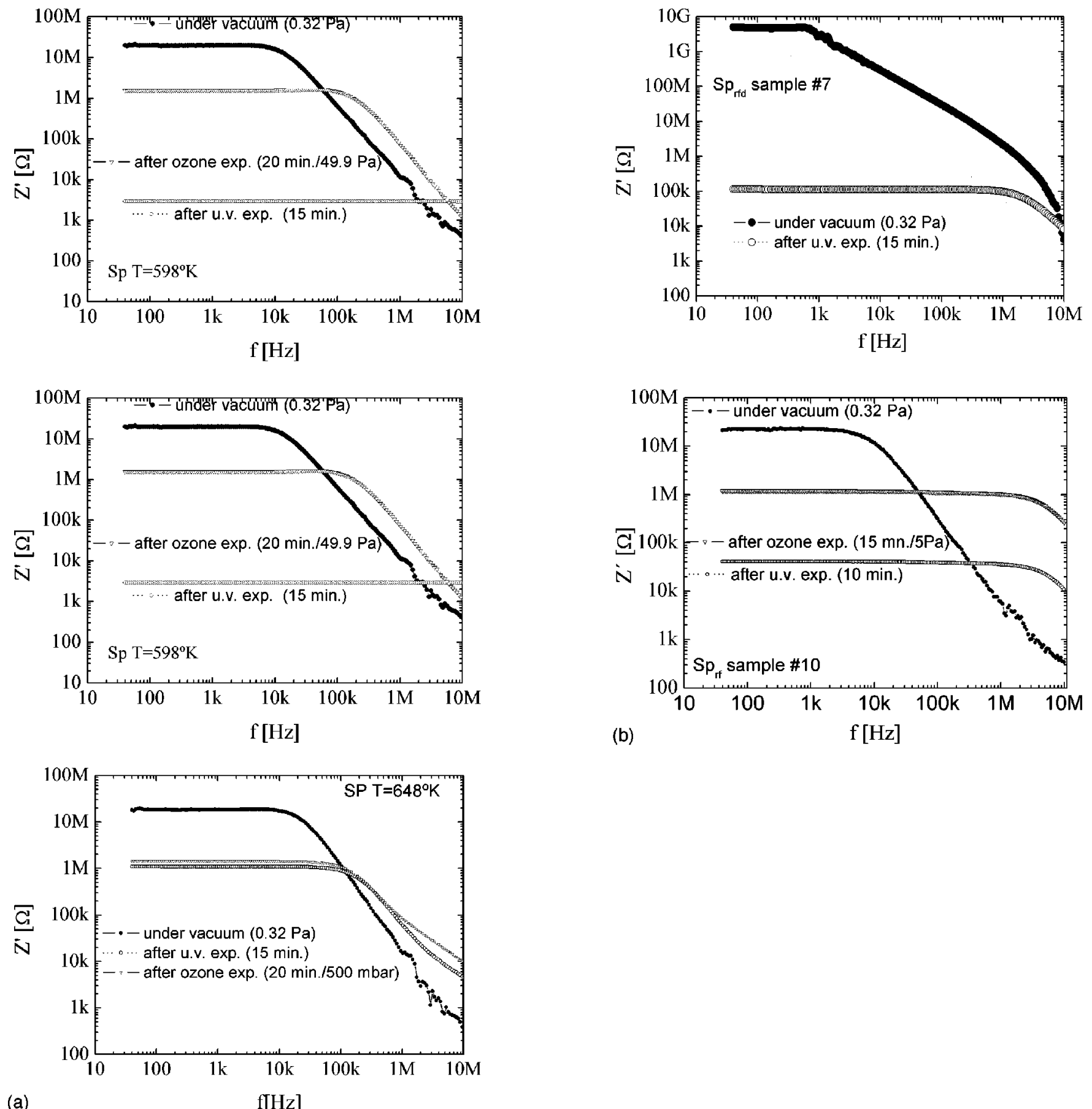

FIG. 3. Dependence of the real component of the ac impedance $\left(Z^{\prime}\right)$ on the frequency for (a) SP films produced at different $T_{s}$ as shown in the inset; (b) Sp rf films produced at room temperature and with grain sizes as shown in the inset.

pen for $S_{\mathrm{UV}}$. In other words, a better ac sensitivity detection is achieved for SP films produced at a low $T_{s}$, which exhibit a more porous and rougher surface.

We have also analyzed the data of the complex electrical modulus $M^{*},{ }^{19,33}$

$$
M^{*}=M^{\prime}-\mathrm{j} M^{\prime \prime}=j \omega C_{0}\left(Z^{\prime}-j Z^{\prime \prime}\right),
$$

where $C_{0}$ is the bulk capacity of a capacitor with the same geometry and under vacuum conditions. By plotting $M^{\prime \prime} / C_{0}$, the data show a peak that moves from low to high frequencies, before and after UV irradiation of the films, as shown in the two plots of Fig. 5. The value of the peak corre- sponds to $1 /(2 C)$ that occur for $f_{\max }=1 / 2 \pi R C$. For this frequency, the maximum of $-Z^{\prime \prime}$ is equal to $R / 2 .^{34,17}$ The frequencies ascribed to $f_{\max }$ coincide with $f_{c}$. Thus, we can consider the cutoff frequency as the maximum relaxation frequency. We can then relate the shift of the peak observed after UV irradiation as corresponding to a change on the electrical mechanism path: from the surface to the bulk, caused mainly by the UV doping effect in compensating vacancies and/or defects in the interfacial region. Our conclusion agrees with what other researchers have obtained in oxide semiconductors, including sintered doped $\mathrm{ZnO}$, where the peak at low frequencies is ascribed 

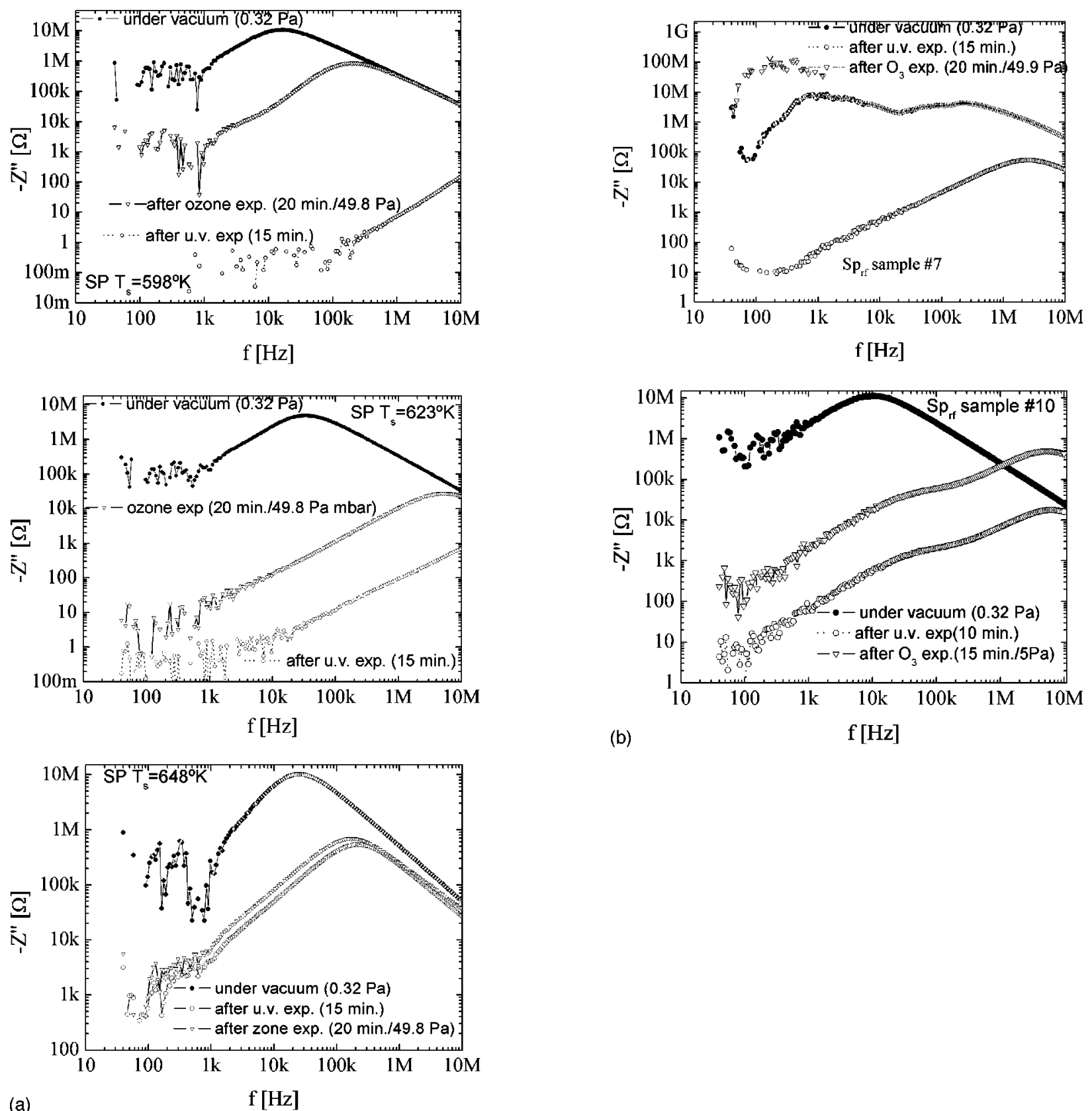

FIG. 4. Dependence of the imaginary component of the ac impedance $\left(-\mathrm{Z}^{\prime \prime}\right)$ on the frequency for (a) Sp films produced at different $T_{s}$ as shown in the inset; (b) $\mathrm{SP}_{\mathrm{rf}}$ films produced at room temperature and with grain sizes of $30 \mathrm{~nm}$ (sample 7) and $55 \mathrm{~nm}$ (sample 10).

to the role of the surface and the peak at high frequencies to the role of the bulk of the grains. ${ }^{17,35}$

The plots of the imaginary part of the total impedance $\left(-Z^{\prime \prime}\right)$ against the real part $Z^{\prime}$ as a parametric function of the frequency ${ }^{17}$ are shown in Fig. 6. The fitting of the experimental points have been obtained by least-mean-square analysis. The intercepts of these semicircles with the real axis give information about the ohmic resistance of the

TABLE I. Characteristic parameters obtained from ac conductivity measurements.

\begin{tabular}{|c|c|c|c|c|c|c|c|c|c|}
\hline \multirow[t]{2}{*}{ Samples } & \multicolumn{3}{|c|}{$-Z^{\prime \prime}$ vs $Z^{\prime}$ plots } & \multicolumn{3}{|c|}{ Imaginary component $\left(-Z^{\prime \prime}\right)$} & \multicolumn{3}{|c|}{ Real component $\mathrm{Z}^{\prime}$} \\
\hline & $f_{\text {peak }}(\mathrm{MHz})$ & $f_{\text {peakUV }}(\mathrm{MHz})$ & $f_{\text {peakO }(\mathrm{MHz})}$ & $S_{\mathrm{UV}}$ & $S_{\mathrm{o}}$ & $f_{\text {peak }(\mathrm{kHz})}$ & $S_{\mathrm{UV}}^{\prime}$ & $S_{\mathrm{O}}^{\prime}$ & $f_{c}(\mathrm{kHz})$ \\
\hline SP325 & 0.016 & 3.800 & 0.210 & $5.5 \times 10^{7}$ & $2.5 \times 10^{5}$ & 15 & $6.7 \times 10^{3}$ & 550 & 15 \\
\hline SP350 & 0.034 & 21.0 & 5.6 & $10^{6}$ & 86 & 24 & $2 \times 10^{3}$ & 11 & 24 \\
\hline SP375 & 0.025 & 0.220 & 0.187 & $10^{4}$ & 1.9 & 22 & 15.5 & 1.2 & 22 \\
\hline $\mathrm{Sp}_{\mathrm{rff}} 7$ & 0.002 & 2.6 & 0.850 & $5.7 \times 10^{5}$ & $6 \times 10^{5}$ & 0.95 & $5 \times 10^{4}$ & $\cdots$ & 0.95 \\
\hline $\mathrm{Sp}_{\mathrm{rf}} 10$ & 0.010 & 6.5 & 5.7 & $1.8 \times 10^{4}$ & 30 & 8.3 & 471 & 28 & 8.3 \\
\hline
\end{tabular}



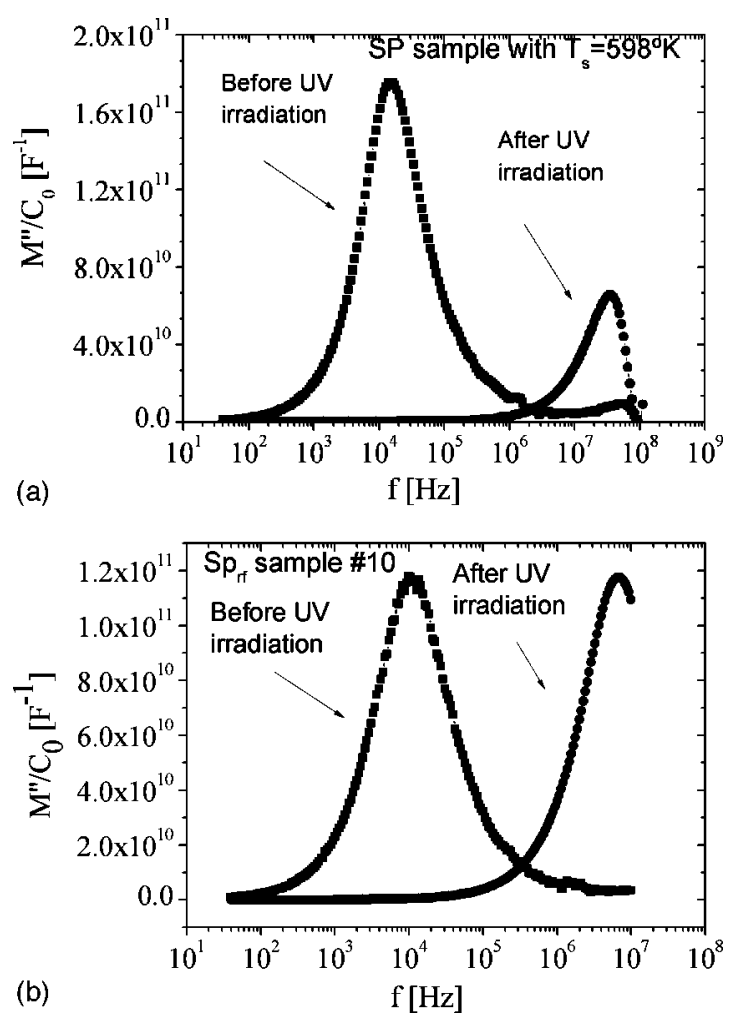

FIG. 5. Dependence of the $\mathrm{M}^{\prime \prime} / \mathrm{C}_{0}$ ratio on the frequency for (a) SP film produced at $598 \mathrm{~K}$; (b) $\mathrm{SP}_{\mathrm{rf}}$ film with grain size of about $55 \mathrm{~nm}$.

system. ${ }^{17}$ For the range of $f$ analyzed, the data are fitted by a semicircle whose shape changes after UV irradiation or film exposure to an ozone atmosphere. This means that, for the frequency range used, the electrodes do not affect the sensor response and so, the main contributions for the impedance come from the bulk and/or surface of the grains. This corroborates our previous assumptions.

The representation of $-Z^{\prime \prime}$ versus $Z^{\prime}$ of the SP films reveal that the semicircles are skewed after the UV irradiation, recovering their original form after exposing the films to an ozone atmosphere. This behavior can be explained by the $\mathrm{ZnO}$ reduction after UV irradiation, with a high number of vacancies compensated by oxygen or $\mathrm{O}_{3}$. This acts as donor dopant, leading to a big change on the transport mechanism path. On the other hand, for the $\mathrm{SP}_{\mathrm{rf}}$ films, the semicircles representing the dependence of $-Z^{\prime \prime}$ on $Z^{\prime}$ are less skewed after UV irradiation. This applies specially to the films with grain sizes below $50 \mathrm{~nm}$. Such behavior could be attributed to a more compact and less rough surfaces of the $\mathrm{Sp}_{\mathrm{rf}}$ films when compared to SP films.

The frequencies $f_{m}$ obtained from the midpoint of the semicircles ${ }^{19}$ are related to the relaxation frequency and so with $f_{c}$. Table I shows the $f_{m}$ values of the films before and after UV irradiation, followed by exposition to an ozone atmosphere (respectively, $f_{m}, f_{m \mathrm{UV}}$ and $f_{m \mathrm{O}}$ ). These data are very close to the ones reported for $f_{c}$. The SP films exhibit the highest $f_{m}$, which increases more than two orders of magnitude after UV irradiation, except for the sample produced at $648 \mathrm{~K}$, which exhibits grains of about $1 \mu \mathrm{m}$ separated by defective layers (excess of defects at the grain boundaries). In this case, the enhancement of $f_{m}$ is only of about one order of magnitude. On the other hand, for the $\mathrm{Sp}_{\mathrm{rf}}$ films, $f_{m}$ is enhanced about three orders of magnitude after UV irradiation. This behavior is consistent with a strong $\mathrm{ZnO}$ reduction promoted by UV irradiation on films made up of nanocrystals and with less defective tissue between grains than the SP films. For the $\mathrm{Sp}_{\mathrm{rf}}$ with grain sizes around 30 , the peak frequency before and after UV irradiation is, respectively, of $2 \mathrm{kHz}$ and $2.6 \mathrm{MHz}$ corresponding to impedances of about $30 \mathrm{M} \Omega$ and $60 \mathrm{k} \Omega$. From both cases we expected an equivalent value for the capacitance of around $10^{-12} \mathrm{~F}$. However, if we take into account that the relative permittivity of $\mathrm{ZnO}$ is around $8-10$, for the gap cell geometry and area $A$ used $\left(1.2 \times 10^{-8} \leqslant A \leqslant 1.2 \times 10^{-9} \mathrm{~m}^{2}\right)$, the steady state capacitance $\left(C=\varepsilon_{0} \varepsilon_{\mathrm{r}} A / d\right)$ should be as high as about $5.6 \times 10^{-17}$ or 5.6 $\times 10^{-16} \mathrm{~F}$. In theory, it should not be compatible with the set of results achieved from the ac impedance measurements. To reach such values, the relative dielectric permittivity should be at least $10^{4}$ times higher than the one of $\mathrm{ZnO}$. To explain these discrepancies as well as the set of impedance results achieved before and after UV irradiation of the undoped $\mathrm{ZnO}$ films analyzed, we propose a simple model based in two phases.

The Arrhenius plot of the dc conductive as a function of the inverse of the absolute temperature (Fig. 7) in the temperature range from 300 to $500 \mathrm{~K}$ for the $\mathrm{Sp}_{\mathrm{rf}}$ films reveals that the main conduction mechanism is by hopping/ percolation. This involves deep and shallow vacancy states located, respectively, at about 1.04 and $0.16 \mathrm{eV}$. The data confirms that the UV irradiation leads mainly to the activation of shallow states, thus bringing about a dopinglike effect that enhances the conductivity in orders of magnitude, function of grain size, and grain boundary defects of the films analyzed.

\section{The model proposed}

In order to interpret the set of results achieved, we propose a simple model as shown in Fig. 8(a). Here, we consider a two phase heterogeneous structure: Phase 1, constituted by the surface grains and the inner boundaries of the aggregation of grains; Phase 2, constituted by the interfacial region between grains and characterized by the existence of a high disorder tissue connecting the different (bulk) grains, similar to what was observed by Schwarz in $\mathrm{ZnO}$ colloid particles when exposed to relative high humidity ${ }^{36}$ or similar to what is observed at the grain boundaries of the metal oxide varistors. ${ }^{37,38}$ Under this condition, subscript 1 will refer to the most resistant phase (the surface of grains, where $\mathrm{d}_{1}$ is the separation between them) that dominates the conduction mechanism before UV irradiation. Subscript 2 will refer to the interfacial contribution to the transport mechanism, ascribed to bulk grains with sizes $d_{2} \gg d_{1}$, distributed along the distance $d$ of the gap cell configuration used. After UV exposure, some vacancies or defect states existing between the grains incorporate $\mathrm{O}_{3}$ atoms. This, in turn, leads to the release of free charge carriers to the boundaries of the interfaces, and consequently to the increase of the films' conductivity, the function of the possible barrier height $\varphi_{b}$ at the 

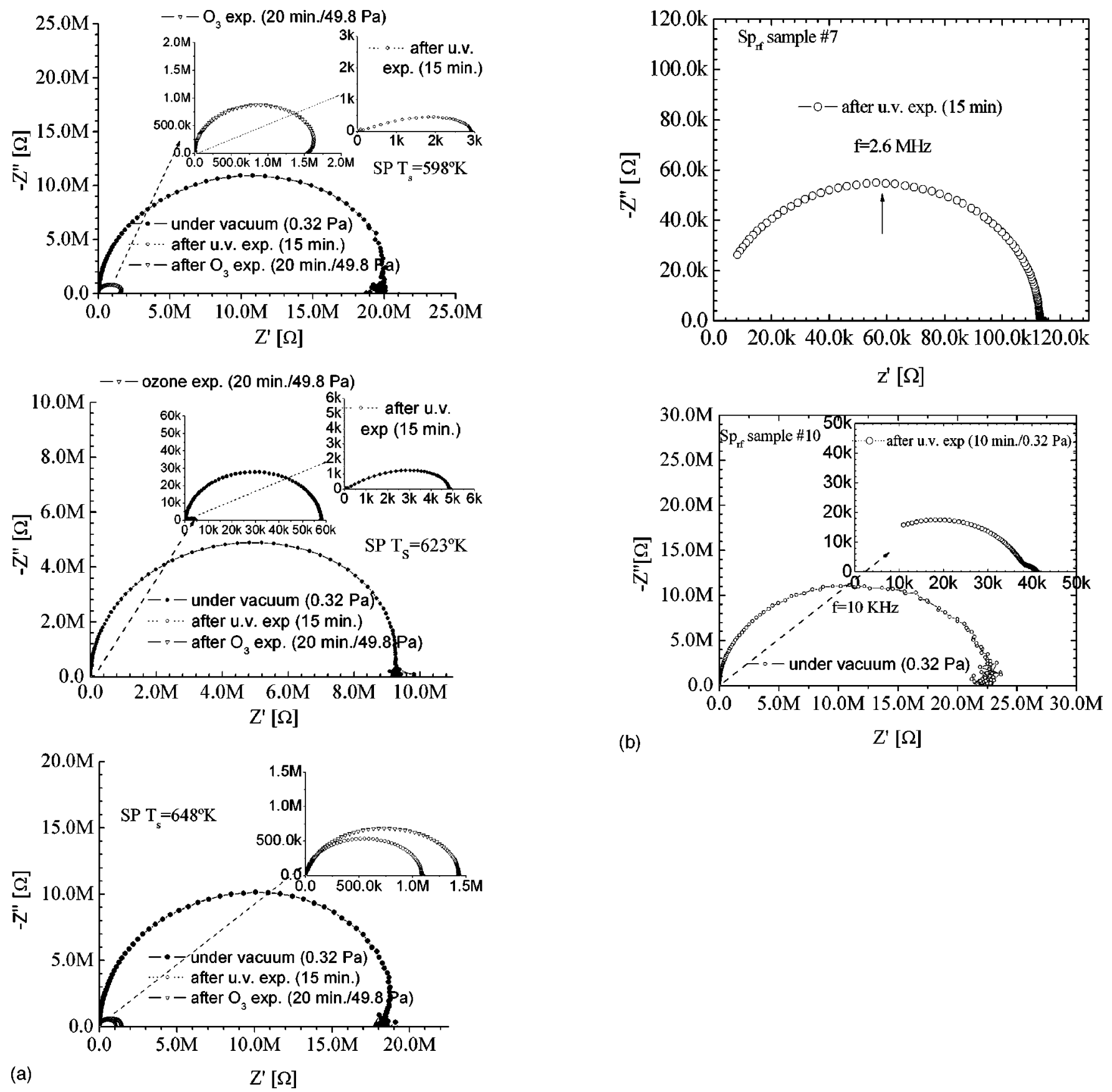

FIG. 6. $-Z^{\prime \prime}$ versus $Z^{\prime}$ for (a) SP films produced at different $T_{s}$ as shown in inset, before UV irradiation, after UV irradiation, and after ozone exposition; (b) $\mathrm{SP}_{\mathrm{rf}}$ films produced at room temperature and with grain size of $30 \mathrm{~nm}$ (sample 7) after UV irradiation and with grain size of $55 \mathrm{~nm}$ (sample 10), before and after UV irradiation.

interfaces and the amount of defects associated with the grain boundaries that may behave as traps for the free carriers. $^{39}$

This model is close to the one where the conductive phase is dispersing in a more resistant matrix. ${ }^{40,41}$ Under this condition, each phase has different conductivities and permittivities, respectively, $\sigma_{1}, \varepsilon_{1}$, and $\sigma_{2}, \varepsilon_{2}$. These can be described by the equivalent electric circuit shown in Fig. 8(b), composed of resistances $R$ and capacitances $C$, whose ideal representation in the complex plane gives rise to at least two semicircles. ${ }^{17}$ The first semicircle appears at low $f$ and corresponds to the role of the surface of the grains, including grain boundaries. The second semicircle corresponds to the role of the bulk of the grains and appears at high frequencies. ${ }^{19}$ The role of contacts (the two extreme resistances) can also influence the shape of curves obtained, when they are not ohmic. ${ }^{42}$ This is not the case under analysis.

Besides that, the presence of defects will affect impedance and capacitance due to the formation of barrier layers at the grain/grain boundary interfaces. However, we have to take into account that this process is averaged over many grain boundaries and is highly difficult to interpret. ${ }^{43}$ Therefore, there are no perfect semicircles but instead deformed arcs whenever a high number of defects/vacancies are present in the structure under analysis. 


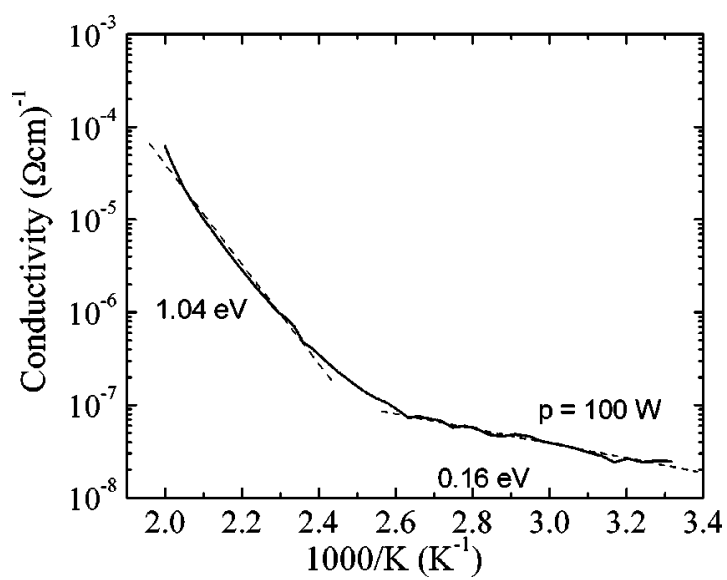

FIG. 7. -dc conductivity versus $T^{-1}$ of an undoped $\mathrm{ZnO}$ film produced by rf magnetron sputtering.

From our experimental data we only notice the existence of one semicircle from the plot of $-Z^{\prime \prime}$ versus $Z^{\prime}$. This behavior has also been observed by other researchers that ascribed it to an electrically inhomogeneous material in which the grain boundary resistance may, in certain circumstances, dominate the overall impedance. ${ }^{19}$

Based on our proposed model, neglecting the effect of the contacts and under static conditions (direct current) we have that $R_{1}=\left(1 / \sigma_{1}\right) \times\left(d_{1} / S\right), R_{2}=\left(1 / \sigma_{2}\right)\left(d_{2} / S\right)$, and $R=R_{1}$ $+R_{2}$. So, the equivalent conductivity of this system can be written as

$$
\sigma=\frac{\sigma_{1} \sigma_{2}}{\sigma_{1} d_{2}+\sigma_{2} d_{1}} d .
$$

Similarly, if we consider the impedance relation of each branch of the equivalent electric circuit $\left[Z_{1}=R_{1} /(1\right.$ $\left.\left.+j \omega R_{1} C_{1}\right), Z_{2}=R_{2} /\left(1+j \omega R_{2} C_{2}\right)\right]$ and that $Z=Z_{1}+Z_{2}, \quad C_{1}$ $=\varepsilon \varepsilon_{1} S / d_{1}, C_{2}=\varepsilon \varepsilon_{2} S / d_{2}$, we reach for the permittivity relations

$$
\varepsilon_{s} \approx \frac{\varepsilon_{1} d_{1} \sigma_{2}^{2}+\varepsilon_{2} d_{2} \sigma_{1}^{2}}{\left(\sigma_{1} d_{2}+\sigma_{2} d_{1}\right)^{2}} \quad \text { (for low frequencies) }
$$

or

$$
\varepsilon_{\infty} \approx \frac{\varepsilon_{1} \varepsilon_{2} d}{\varepsilon_{1} d_{2}+\varepsilon_{2} d_{1}} \quad \text { (for high frequencies) }
$$

where the frequency $f_{m}$ for which the relaxation occurs depends also on the characteristics of the phases present and is given by

$$
f_{m} \cong \frac{1}{2 \pi \varepsilon_{0}} \frac{\sigma_{1} d_{2}+\sigma_{2} d_{1}}{\varepsilon_{1} d_{2}+\varepsilon_{2} d_{1}}
$$

where $\varepsilon_{0}$ is the vacuum permittivity and $d=d_{1}+d_{2}$. We assume that $d_{1}$ has dimensions of the order of the intergrain or porous space, ranging from a few tenths of nanometers to thousands of nanometers. The relaxation time of each of the phases is given by $\tau_{1} R_{1} C_{1}=\varepsilon_{0} \varepsilon_{1} / \sigma_{1}$ and $\tau_{2}=R_{2} C_{2}=\varepsilon_{0} \varepsilon_{2} / \sigma_{2}$, where the maximum peak position in the semicircles is related $f_{\max }$, as described above. ${ }^{28}$

To test the proposed model, we must fit it with the set of experimental data obtained. For instance, for the $\mathrm{Sp}_{\mathrm{rf}}$ film constituted by small nanocrystals we saw that the equivalent capacitance extracted from the impedance measurements is of about $10^{-12} \mathrm{~F}$, compatible with $\varepsilon_{\mathrm{r}} \sim 10^{5}$. Apart from that, we also saw that if we take into account that the permittivity in both phases is almost equal to 8 , the only way to explain the behavior observed is by looking at differences between the conductivity in the two phases and at the $d / d_{1}$ ratio. If we consider that, after UV irradiation, the main conduction

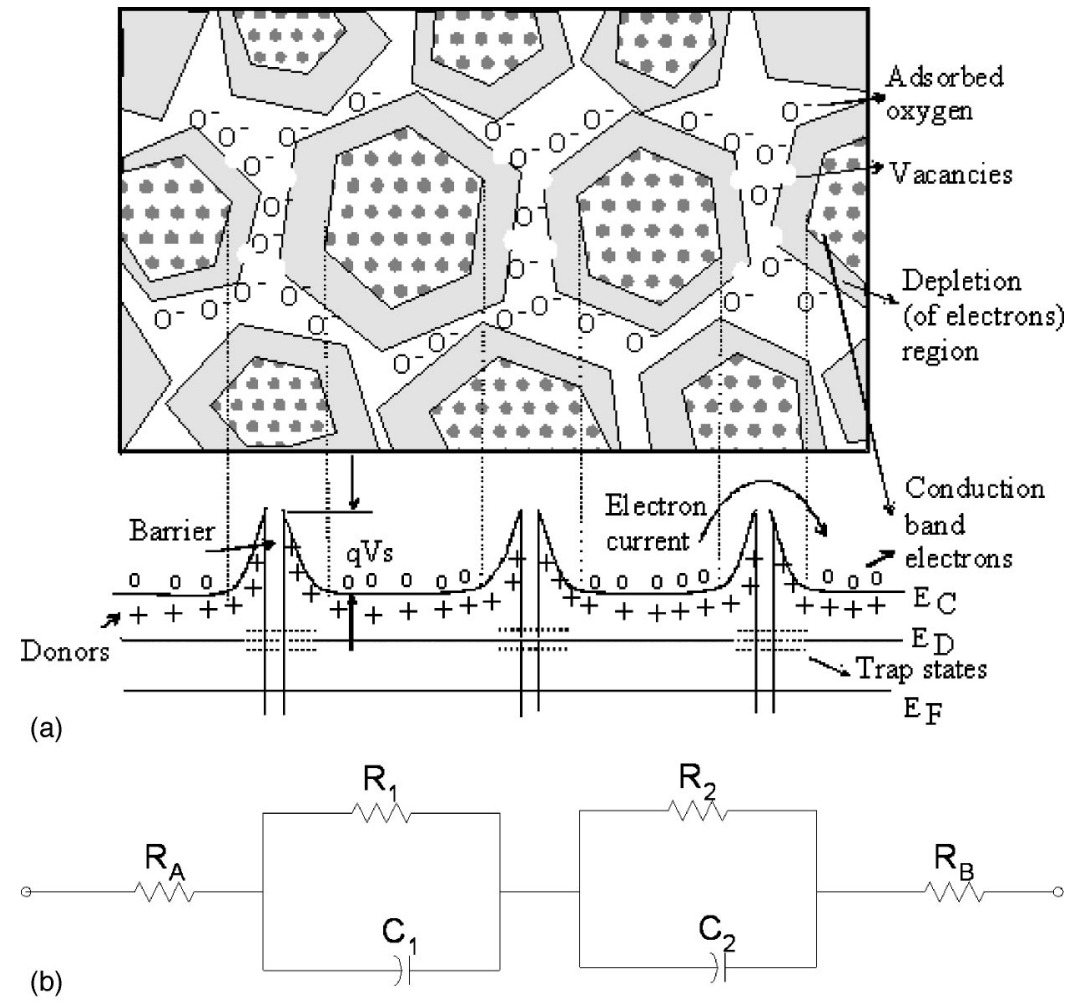

FIG. 8. (a) Schematic model of the microstructure and corresponding energy bands of an oxide semiconductor where $E_{c}$ is the button of the conduction band energy level, $E_{d}$ is the energy level of the possible donor states, $E_{f}$ is the Fermi energy level, and $\varphi_{b}$ is the potential barrier in the interfacial region. The open circles between grains correspond to (empty) vacancies that can incorporate $\mathrm{O}_{3}$ atoms that act as a donorlike impurity. (b) Sketch of the equivalent electric circuit, where the first block tries to visualize the role of surface grains and the second block the role of the interfacial region and the bulk of the grains, according to a two-phase electrical model. 


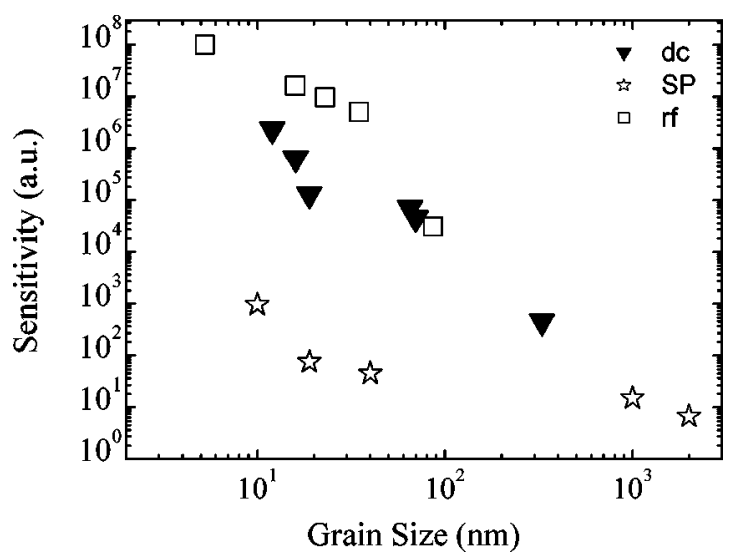

FIG. 9. Dependence of the $\mathrm{ZnO}$ sensitivity deposited by various techniques on the average grain size (measured by AFM) of the films.

mechanism involves the bulk of the grains due to the role of the phase ascribed to the interfacial region, we can estimate $\sigma_{2} \sim 20 \Omega^{-1} \mathrm{~m}^{-1}$. Substituting $\sigma_{1}$ and $\sigma_{2}$ in Eq. (3a) we obtain $d / d_{1} \sim 10^{4}$.

Now, if we take into account the size of the nanocrystals $(30 \mathrm{~nm})$ this means that between the contacts we may have about $5 \times 10^{4}$ grains, assuming that the grains make a continuous chain. Nevertheless, we do not have a continuous chain of grains, since we have interfacial spaces or even nanoporous separating the grains, with sizes up to $10-20 \mathrm{~nm}$, as revealed by the SEM or AFM micrographs. Therefore, we will arrive to values of $d / d_{1}$ very close to the ones obtained by using the model proposed.

The same type of analysis was done for the other set of samples studied, where the proposed model fits well with the set of experimental points obtained. There, we notice that the $d / d_{1}$ ratio decreases either when the grain size increases or when the dimension of the interfacial (porous) also increases, approaching 15 for the SP $\mathrm{ZnO}$ films. In other words, the $\mathrm{d} / \mathrm{d}_{1}$ ratio can be considered as the dielectric amplification factor.

\section{DISCUSSION OF THE RESULTS}

The analysis of the dc sensitivity dependence on the grain size (Fig. 9) shows that the films with the largest grains have the poorest performances in terms of ozone sensing. In general, films deposited by SP exhibit dc sensitivities up to $7 \times 10^{3}$, while the sputtered films exhibit sensor sensitivities between $10^{3}$ and $10^{8}$, as the grain size changes from 120 to $4 \mathrm{~nm}$. This means that the sensitivity increases as the grain size decreases. The highest value was achieved for highly textured $\mathrm{ZnO}$ films deposited by rf sputtering technique at room temperature.

The differences in the behaviors observed for the two types of sputtered films (dc and rf) are attributed to differences in the grain sizes, the degree of the films' compactness, and the surface morphology, highly dependent on $T_{s}$ used. On the other hand, the poor dc sensitivities achieved in the films deposited by SP are attributed to its porosity, surface roughness, and surface contamination. These characteristics do not exist in the films produced by the sputtering technique. Nevertheless, the experimental data show that the state of surface oxidation, together with the degree of porosity and surface roughness of the SP films, makes these films highly ac sensitive to UV or ozone exposition, agreeing quite well with the model proposed. Indeed, the experimental data show that SP films with grain sizes in the range from 40 to $100 \mathrm{~nm}$ reveal very high $S_{\mathrm{UV}}$ and $S_{\mathrm{O}}$ values, higher than the ones corresponding to their $S_{\mathrm{UV}}^{\prime}$ and $\mathrm{S}_{\mathrm{O}}^{\prime}$ counterparts.

The overall enhancement observed on the dc and ac conductivities of the films analyzed is attributed to the absorption of photons with energy higher than the energy band gap of the $\mathrm{ZnO}$ that lead to the generation of electron-hole pairs by light absorption. Furthermore, oxygen is desorbed from the surface of the sample, leading to the creation of free electrons ${ }^{44}$ as translated by Eqs. (5). Following that, the films subsequently exposed to ozone at room temperature exhibit a strong increase in resistance caused by reoxidation, function of the state of the surface of the $\mathrm{ZnO}$ films [see Eq. (6)]. That is, the main mechanism responsible for large conductive changes in $\mathrm{ZnO}$ films is the formation and annihilation of oxygen vacancies when the film sustains an UV irradiation whose associated energy is higher than the bonding energy between $\mathrm{Zn}$ and $\mathrm{O}$. This leads to $\mathrm{O}$ release from the film in the gaseous form, leaving behind two valence electrons in the vacant site. If both electrons remain in the vacancy, the neutrality charge is preserved and the vacancy has 0 effective charges. If one or both of the localized electrons are excited and transferred away from the vacancy, the vacancy is left with an effective positive charge. The charged oxygen vacancy (singly or doubly ionized) becomes an electron- trapping site where one or two electrons can be made available for conduction. The formation of doubly charged oxygen vacancies can be described by the defect equation ${ }^{45}$ applied to $\mathrm{ZnO}$ when exposed to UV light:

$$
\begin{aligned}
& \left(\mathrm{Zn}^{2+} \mathrm{O}^{2-}\right)_{\text {surf }}+(h v)_{\mathrm{UV}} \rightarrow\left(\mathrm{Zn}^{2+} \mathrm{O}^{-}\right)_{\text {surf }}+e, \\
& \left(\mathrm{Zn}^{2+} \mathrm{O}^{-}\right)_{\text {surf }}+(h v)_{\mathrm{UV}} \rightarrow \mathrm{Zn}^{2+}+\mathrm{O}_{\text {surf }}+2 e, \\
& 2 \mathrm{O}_{\text {surf }} \rightarrow \mathrm{O}_{2}(g) .
\end{aligned}
$$

The subsequent oxidation in ozone leads to annihilation of the charged oxygen vacancies by incorporation of oxygen into the film by surface recombination, leading to

$$
\left(\mathrm{Zn}^{2+} \mathrm{O}^{-}\right)_{\text {surf }}+\mathrm{O}_{3} \rightarrow\left(\mathrm{Zn}^{2+} \mathrm{O}^{2-}\right)_{\text {surf }}+\frac{3}{2} \mathrm{O}_{2}(g) .
$$

Thus, the charge carrier concentration decreases drastically.

This photoreduction and subsequent oxidation procedure, also characteristic of other oxides such as $\operatorname{In}_{2} \mathrm{O}_{3}$, ${ }^{16}$ is fully reversible and can be repeated many times (Fig. 10). The dc sensitivity covers a range of more than eight orders of magnitude, depending on the size of the crystallites, degree of film compactness, and state of surface oxidation. This type of behavior can be clearly explained by our two-phase proposed model, and the set of recorded electrical experimental data also fit well.

The data achieved lead to film's dc and ac sensitivity to the UV irradiation, exhibiting values at least two orders of magnitude larger than the results achieved with $\operatorname{In}_{2} \mathrm{O}_{3}$ (Ref. 16) films. In addition, the dc response time is about one order of magnitude faster than the recovering time. 


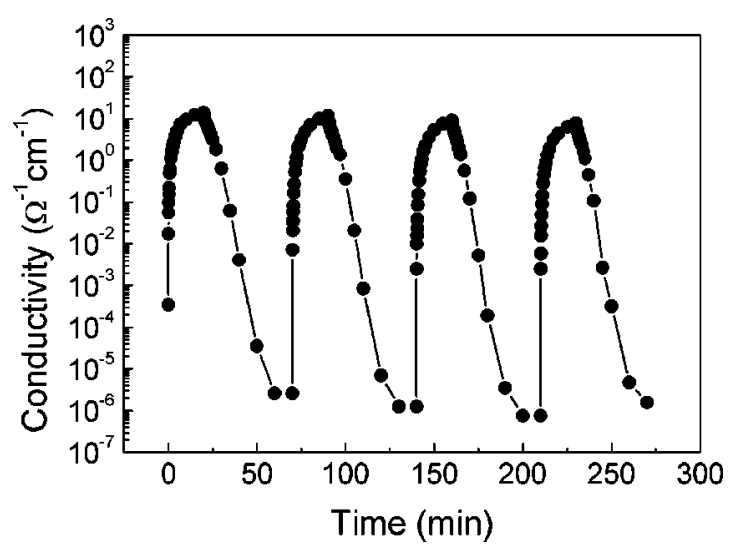

FIG. 10. Photoreduction and reoxidation cycles of a rf sputtered undoped $\mathrm{ZnO}$ film produced at room temperature.

The ac conductivity data also reveals that the films with high roughness and porosity (SP) when constituted by large nanocrystals (produced at low $T_{s}$ ) have an excellent reactance response (of more than seven orders of magnitude), with $f_{\text {peak }} \approx 15 \mathrm{kHz}$. On the other hand, the sputtering films with similar grain sizes but with smoother surfaces exhibit reactance sensitivities of about two orders of magnitude smaller than the one of SP films.

The behavior of the sensor response can be understood by taking into account the surface dependence on the effects involved and the model proposed. In films with large and porous grains, the area of the grain boundaries and the type of defects ascribed to them control the conduction mechanism.

When the grain size decreases, the area of grain boundaries will increase. Therefore, the absorption and desorption of atoms from the surface has a much larger influence on the conductivity of the sample because the depletion layer caused by trapped charge carriers occupies a large fraction of the grain surface/volume. Consequently, the conductivity changes observed during UV irradiation and reoxidation of the films are strongly dependent on surface morphology and type of contaminants incorporated in surface states. This is mainly the case for films constituted by large grain sizes according to our model and confirmed by ac measurements. On the other hand, for films with clean and smooth surfaces, such as the ones produced either by dc or rfs puttering, the dimension of the grains, film compactness, and surface texture are of primordial importance in determining the lateral surface through which the gas is absorbed. These factors can lead to drastic changes in the film's conductivity and equivalent capacitance, especially when films are porous (SP films produced at $T_{s}<598 \mathrm{~K}$ ). These data agree closely with the model proposed in this paper and confirmed by the set of dc and ac impedance measurements performed.

\section{CONCLUSIONS}

To summarize our work, we have demonstrated the ability to use $\mathrm{ZnO}$ thin films produced by different techniques, as ozone sensors at room temperature, after being exposed to UV irradiation. The results achieved show that the dc and ac behavior of the films before and after UV irradiation are dependent on the type of structure produced. The highest dc sensitivity (above $10^{5}$ ) reaches values ranging from 5 to $45 \mathrm{~nm}$, with an uncontaminated texture surface. This is the case of films produced either by dc sputtering at low $T_{s}$ or by rf sputtering at room temperature. In contrast, films with a polycrystalline structure with large grain sizes and with contaminated surfaces, such as the ones produced by SP, have the lowest dc sensitive values. It can be deduced from the experimental work shown in this paper that the best ac sensitivity (exceeding 105) was obtained in SP films with low grain sizes (below $100 \mathrm{~nm}$ ) and highly porous produced at low substrate temperatures that exhibit relaxation frequencies above $15 \mathrm{kHz}$.

\section{ACKNOWLEDGMENTS}

This research was supported by the European Commission through the TMR program HAFAM (Contract No. FMRX-CT97-0141). The Portuguese authors would also like to thank the financial support given by "Fundação para a Ciência e a Tecnologia" (Foundation for Science and Technology) through a contract with CENIMAT, and the Project No. POCTI/CTM/38924/2001, and to R. Igreja for the fruitful discussions and help given during the ac impedance measurements.

${ }^{1}$ P. T. Moseley, Meas. Sci. Technol. 8, 223 (1997).

${ }^{2}$ Y. Xu, X. Zhou, and O. T. Sorensen, Sens. Actuators B 65, 2 (2000).

${ }^{3}$ R. A. Robie, P. M. Bethke, and K. M. Beardsley, in Handbook of Chemistry and Physics, 83rd ed., edited by D. R. Lide (CRC, Boca Raton, 2002), Vol. 1, Chap. 12, p. 105.

${ }^{4}$ P. F. Carcia, R. S. McLean, M. H. Reilly, and G. Nunes, J. Appl. Phys. 82, 1117 (2003).

${ }^{5}$ H. Takikawa and K. Kimura, Thin Solid Films 74, 377 (2000).

${ }^{6}$ S. N. Bai and T. Y. Tseng, J. Appl. Phys. 74, 1, (1993); 74, 695 (1993).

${ }^{7}$ E. Fortunato et al., Adv. Eng. Materials 4, 610 (2002).

${ }^{8}$ J. Xu, Y. Shun, Q. Pan, and J. Qin, Sens. Actuators B 66, 161 (2000).

${ }^{9}$ M. Aslam, V. A. Chaudhary, I. S. Mulla, S. R. Sainkar, A. B. Mandale, A.

A. Belhekar, and K. Vijayamorhanan, Sens. Actuators B 75, 162 (1999).

${ }^{10}$ M. Kudo, T. Kosaka, Y. Takahashi, H. Kokusen, N. Sotani, and S. Hasegawa, Sens. Actuators B 69, 10 (2000).

${ }^{11}$ G. Sberveglieri, Gas Sensors Principles, Operation and Developments (Kluwer Academic, Dordrecht, 1992).

${ }^{12}$ G. M. Chow, I. A. Ovid'ko, and T. Tsakalakos, in Nanostructured Films and Coatings, NATO Advanced Studies Institute Series Vol. 78 (Kluwer, Dordrecht, 2000).

${ }^{13}$ T. Takada, K. Suzuki, and M. Nakane, Sens. Actuators B 13-14, 404 (1993).

${ }^{14}$ M. Z. Atasshbar, B. Gong, H. T. Sun, W. Wlodarsk, and R. Lamb, Thin Solid Films 354, 162 (1999).

${ }^{15}$ C. Xirouchaki, K. Moschovis, E. Chatzitheodoridis, G. Kiriakidis, H. Boyce, and P. Morgen, J. Electroch. Mat. 28, 26 (1999).

${ }^{16}$ M. Bender, N. Katsarakis, E. Gagaoudakis, E. Hourdakis, E. Douloufakis, V. Cimalla, and G. Kiriakidis, J. Appl. Phys. 90, 5382 (2001).

${ }^{17}$ F. A. Grant, J. Appl. Phys. 29, 1 (1958); 29, 76 (1958).

${ }^{18}$ M. A. Alim, M. A. Seitz, and R. W. Hirthe, J. Appl. Phys. 63, 7, (1988); 63, 2337 (1988).

${ }^{19}$ M. Andres-Verges and A. R. West, J. Electroceramics 1, 2 (1993); 1, 125 (1993).

${ }^{20}$ J. T.S. Irvine, D. C. Sinclair, and A. R. West, Adv. Mater. 2, 3 (1990); 2, 132 (1990).

${ }^{21}$ M. A. Alim, J. Am. Ceram. Soc. 72, 1 (1989); 72, 28 (1989).

${ }^{22}$ B. S. Chiou, and M. C. Chung. J. Electron. Mater. 20, 10 (1991); 20, 885 (1991).

${ }^{23}$ G. D. Mahan, L. M. Levinson, and H. R. Phillipp. J. Appl. Phys. 50, 2779 (1979).

${ }^{24}$ D. C. Sinclair and A. R. West. J. Appl. Phys. 66, 3850 (1989).

${ }^{25}$ P. Nunes, B. Fernandes, E. Fortunato, P. Vilarinho, and R. Martins, Thin 
Solid Films 337, 176 (1999).

${ }^{26}$ P. Nunes, D. Costa, E. Fortunato, and R. Martins, Vacuum 64, 293 (2002)

${ }^{27}$ G. Kiriakidis, N. Katsarakis, M. Bender, E. Gagaoudakis, and V. Cimalla, Mater. Phys. Mech. 1, 83 (2000).

${ }^{28}$ W. G. Wyckoff, Crystal Structures, 2nd ed. (Wiley, New York 1968) Vol 4, p. 528 .

${ }^{29}$ J. C. Lee, K. H. Kang, S. K. Kim. K. H. Yoon, I. J. Park, and J. Song, Sol. Energy Mater. Sol. Cells 64, 185 (2000).

${ }^{30}$ P. Scherrer, Nachr. Ges. Wiss. Goettingen, Math.-Phys. Kl. 2, 96 (1918).

${ }^{31}$ P. Nunes, F. M. Braz Femandes, R. J.C. Silva, E. Fortunato, and R. Martins, Key Eng. Mater. 230-232, 599 (2002).

${ }^{32}$ S. Roy, and S. Basu, Bull. Mater. Sci. 25, 6, (2002); 25, 513 (2002).

${ }^{33}$ I. M. Hodge, M. D. Ingram, and A. R. West, J. Electroanal. Chem. 74, 2 , (1976); 74, 125 (1976).

${ }^{34}$ I. M. Hodge, M. D. Ingram, and A. R. West, J. Electroanal. Chem. 58, 2 (1975); 58, 429 (1975).

${ }^{35}$ A. Smith, J. F. Baumard, P. Abelard, and M.-F. Denanot, J. Appl. Phys.
65, 5119 (1989).

${ }^{36}$ G. Schwarz, J. Phys. Chem. 66, 3636 (1962).

${ }^{37}$ P. R. Bueno, E. R. Leite, M. M. Oliveira, M. O. Orlandi, and E. Longo, Appl. Phys. Lett. 79, 1 (2001); 79, 48 (2001).

${ }^{38}$ S. N. Bai, and T. Y. Tseng, J. Electron. Mater. 21, 11 (1992); 21, 1073 (1992).

${ }^{39}$ S. R. Morrison, in Semiconductors Sensors, edited by S. M. Sze, (Wiley, New York, 1994) Chap. 8.

${ }^{40}$ C. Weaver, Adv. Phys. 11, 83 (1962).

${ }^{41}$ L. K.H. van Beeck, Prog. Dielectr. 7, 69(1967),

${ }^{42}$ E. Hartmann, Cryst. Res. Technol. 16, 8 (2001); 16, 911 (2001).

${ }^{43}$ J. Fleig and J. Maier, J. Eur. Ceram. Soc. 19, 693 (1999).

${ }^{44}$ Y. Takahashi, M. Kanamori, A. Kondoh, H. Minoura, and Y. Ohya, Jpn. J. Appl. Phys., Part 1 33, 6611 (1994).

${ }^{45}$ P. Kofsad, Nonstoichiometry, Diffusion and Electrical Conductivity in Binary Metal Oxides (Wiley, New York, 1972). 